\title{
Metabolites: novel therapeutics or "me-too" drugs? Using desvenlafaxine as an example
}

\author{
Thomas L. Schwartz* \\ Department of Psychiatry, SUNY Upstate Medical University, Syracuse, New York, USA
}

Received 30 May 2012; Accepted 1 July 2012

Key words: Antidepressants, desvenlafaxine, major depressive disorder, metabolites, pharmacokinetics, venlafaxine.

This issue of CNS Spectrums hosts an article that discusses the use of long-term and high-dose desvenlafaxine for treating major depressive disorder (MDD) and a meta-analytic evaluation of this novel, slowrelease metabolite versus its parent compound, the stalwart antidepressant, venlafaxine ER. Over the last decade, the rising cost of healthcare has created debates over the lack of a blockbuster antidepressant pipeline. Clinicians have seen the emergence of drugs that are metabolites, isomers, and slow-release preparations that are placed into the market as new antidepressant entities, which are highly criticized as being "patent extenders" or "me-too" drugs that really offer no clear advantage per the lay press and pharmaceutical company critics.

Clinicians are often asked why they would choose a newer, more expensive drug over an often used, inexpensive agent, especially in the wake of federally funded, sweeping studies such as STAR ${ }^{1}{ }^{1}$ or CO-MED ${ }^{2}$ that seem to foster the idea that there may be no difference regarding antidepressant agents chosen in regards to outcome, supporting the notion that cheaper generic antidepressants would be cost-effective and frontline treatment as such. The data in the Coleman et al. meta-analysis in this journal issue would suggest that the newer metabolite, desvenlafaxine, offers a safety benefit of statistically less nausea, but the story and potential benefit of desvenlafaxine may be more complicated and may have to translate from the theoretical and preclinical into the real world of prescribing to depressed patients.

Unequivocally, the brand name metabolite antidepressant, desvenlafaxine, has less CYP450 2D6 inhibition and is less of a substrate as well. ${ }^{3}$ Less in these pharmacokinetic areas should equal fewer side effects, as patients should metabolize and process desvenlafaxine more efficiently, avoiding toxicity-induced adverse

*Address for correspondence: Thomas L. Schwartz, Associate Professor, Director of Adult Clinical Services, Dept. of Psychiatry, SUNY Upstate Medical University, Syracuse, NY 13210, USA.

(Email: schwartt@upstate.edu) effects. Preskorn ${ }^{3}$ further suggests that observing the metabolism of venlafaxine into desvenlafaxine is an optimal way to study dose-response curves in patients, as conversion of venlafaxine to desvenlafaxine is $100 \%$ dependent and isolated to the CYP450 2D6 isoenzyme system. Patients with normal concentrations of CYP450 2D6 hepatic enzymes have no difficulty converting venlafaxine into desvenlafaxine, both of whose levels may be easily measured. Ultimately these efficient, normal patients will show very high levels of desvenlafaxine and minimal to no venlafaxine parent drug after absorption through the gut. A ratio of desvenlafaxine (DES) to venlafaxine (VEN) will almost always be greater than one. Those who are CYP450 2D6-deficient genetically will have ratios less than one, as they cannot easily convert venlafaxine to desvenlafaxine. In fact, Nichols et al. ${ }^{4}$ supportively discovered that efficient patients had $400 \%$ more ability to convert venlafaxine to desvenlafaxine.

Why is this important when comparing parent drug to metabolite clinically for those conducting a psychopharmacology practice? Lobello et al. ${ }^{5}$ conducted a retrospective analysis of venlafaxine trials and found that patients with adequate CYP450 2D6 had better venlafaxine antidepressant responses compared to enzyme-poor patients. These latter patients could not convert parent drug, venlafaxine, into the active antidepressant metabolite, desvenlafaxine. Extrapolating, it appears that the ability to convert venlafaxine to desvenlafaxine, with a ratio greater than one affords a greater likelihood of antidepressant response. In this way, the two products are fundamentally different.

Should clinicians obtain a blood sample in the office and genotype patients to determine their CYP450 2D6 status? Definitively, poor metabolizers should not be placed on the parent drug, venlafaxine, and should be on the more expensive metabolite, desvenlafaxine, as these patients can now actually respond to the antidepressant they have been prescribed. This makes the newer, desvenlafaxine agent worth it clinically and not a "me too" or "patent-extending" drug, as there is a 
clear difference in this particular population. This may not be a dose-response curve but rather a differential response rate between these two agents in two distinct populations. Statistically the drugs are similar except for nausea, but to CYP450 2D6-poor patients, there might be a remarkable $100 \%$ difference in that they could waste weeks to months trying the generic parent drug first if they are not genotyped beforehand.

Another pharmacokinetic difference between the parent drug and the metabolite is regarding patient expression of P-glycoprotein (P-gp). P-gp creates barriers within the human body, i.e., blood-brain barrier, placental barrier, etc., that are responsible for allowing xenobiotics, toxins, medications into or out of compartmentalized body spaces. Increased expression of P-gp in cell membranes will increase transport within the blood-brain barrier for example. It is counterintuitive, but increased transport here means exiting drugs out of brain tissue. P-gp is located on capillary endothelial cells in the brain, and active P-gp molecules efflux drugs, toxins, and proteins away from the brain and back into systemic circulation. Venlafaxine is a known inducer of the MDR1 gene, which codes for P-gp. Greater levels of P-gp expression are often associated with greater drug efflux (a greater barrier) away from the brain, thus lowering its central bioavailability. P-gp's greatest influence on drug metabolism may occur at the level of the liver hepatocyte where drugs or toxins must cross into the hepatocyte to be able to activate further CYP450-based metabolism or degradation. Venlafaxine induced P-gp expression here may prevent hepatic access and conversion to desvenlafaxine. Plasma venlafaxine drug levels now remain increased, posing a risk of ongoing side effects, while desvenlafaxine levels are low, likely inhibiting a clinical response. Bachmeier et al. ${ }^{6}$ suggests that venlafaxine induces P-pg expression twofold over desvenlafaxine in vitro, supporting the theories above and again suggesting that the two drugs are fundamentally different.

P-gp also is utilized in the gut to lower absorption into hepatic circulation. Drugs that are substrates for P-gp are more likely to not be absorbed, lowering their clinical utility. A better profile may exist for the desvenlafaxine metabolite, as it is not a P-gp substrate and may be more easily absorbed. For example, there would not be competition for absorption in the medically ill MDD patient who is simultaneously taking other P-gp inducers or substrates, such as nelfinavir, amprenavir, saquinavir, digoxin, verapamil, ketoconazole, quinidine, talinolol, dexamethasone, cyclosporine, St. John's Wort, sertraline, paroxetine, citalopram, amitriptyline, nortriptyline, etc., thus allowing better drug plasma levels and chance of antidepressant response. 7,8
Summarizing, there should be a distinct minority of patients for whom the metabolite antidepressant desvenlafaxine is safer and better tolerated. This editorial could end with a simple, data-driven answer: desvenlafaxine should be used only in those patients who (a) are sensitive to nausea-based side effects (up to $8 \%$ per venlafaxine ER clinical trials outlined in its FDA package insert), (b) have clear CYP450 2D6-poor metabolizer status $\left(10 \%\right.$ of Caucasians $\left.{ }^{9}\right)$, or (c) are P-gp efficient with more $\mathrm{C}$ alleles of the human MDR1 gene, allowing less transport of the drug from gut to liver and liver to systemic circulation $\left(40 \%\right.$ of Caucasians $\left.{ }^{10}\right)$. Therefore, the newer brand name metabolite antidepressant would theoretically be better absorbed in active form, maintaining safe and reasonable plasma levels with actual and theoretical supporting data for $8-40 \%$ of patients, depending on individual genetic variables, when compared to the parent, inexpensive generic antidepressant.

If this theorizing is too grandiose, then the practitioner may not need this extensive knowledge, but should understand that the parent drug venlafaxine is metabolized into desvenlafaxine. Given this factual premise, patients on the parent drug will have two active drugs and their respective side effects in their systems. A more interesting clinical study might be to investigate those patients who develop side effects of any kind on the parent drug and switch them over to the new metabolite, as this is a common strategy in clinical practice. The metabolite in these specific cases might be better tolerated, as there is now one drug entity in systemic circulation and not two. Guico-Pabia et al. ${ }^{11}$ observed patients who had just completed a trial of placebo versus venlafaxine ER versus desvenlafaxine. Nonresponders to this acute phase were switched from placebo onto desvenlafaxine, venlafaxine onto desvenlafaxine, or continuation on desvenlafaxine. There were no clear differences in safety or efficacy between these groups. One would expect fewer side effects if patients were switched from the parent drug onto the metabolite, but this did not occur. A confounding variable here was that desvenlafaxine was dosed at 200-400 mg per day, when the usual clinical dose is $50 \mathrm{mg} / \mathrm{d}$ per regulatory guidelines. High starting doses are associated with poorer tolerability. A slower, flexible titration schedule mimicking those used in clinical practice is likely warranted. Also, patients on the parent drug who had serious adverse effects were often dropped from the study prior to the opportunity to be switched to the theoretically safer metabolite. A better study would be to switch patients at the time of parent drug side effect occurrence in hopes of avoiding a study drop-out due to the parent drug. This type of study has not been published.

This begs a second question: Are the doses of venlafaxine and desvenlafaxine equivalent? The 
desvenlafaxine range in the U.S. is $50-400 \mathrm{mg} / \mathrm{d}$. A majority of published materials now discusses the $50 \mathrm{mg}$ dose, and corporate data suggest that 50 or $100 \mathrm{mg}$ are the most often used daily doses. This journal issue has now published a higher dose study (Ferguson et al.) in order to remind the reader that higher doses have been studied and to provide education about the safety and utilization of higher antidepressant dosing.

Regarding desvenlafaxine dosing, Ferguson et al. might suggest that higher doses have similar side effects to lower doses, albeit that occur now at higher frequencies. This makes intuitive sense, as most drugs will have more side effects as they are dose escalated, but is there merit in regard to desvenlafaxine dose escalating? Bech et al. ${ }^{12}$ retrospectively analyzed desvenlafaxine trials and compared outcomes across doses ranging from $50-400 \mathrm{mg} / \mathrm{d}$. Interestingly, all doses were effective, but there were no apparent advantages to the higher doses. Treatment effect sizes were similar across all dose ranges. Based on this type of analysis, regulatory wording regarding desvenlafaxine dosing suggests "no additional benefit" to doses greater than $50 \mathrm{mg} / \mathrm{d}$. Therefore, both the top-down view and evidence base would suggest that dose escalating is not warranted.

That brings us back to dose equivalencies in venlafaxine/desvenlafaxine dyad. The minimum therapeutic dose of venlafaxine ER is $75 \mathrm{mg} / \mathrm{d}$, whereas that of desvenlafaxine is $50 \mathrm{mg} / \mathrm{d}$. Of note, all therapeutic doses ever studied of the latter $(50-400 \mathrm{mg} / \mathrm{d}$ ) have been statistically efficacious. There has not been a minimum therapeutic dose discovered for desvenlafaxine. The first interpretation should clinically state that $75 \mathrm{mg}$ must equal $50 \mathrm{mg}$ based upon regulatory trials and FDA benchmarks. A pharmacodynamic interpretation would differ in that $75 \mathrm{mg}$ of venlafaxine ER is likely a pure serotonin reuptake inhibitor (SRI) with little norepinephrine reuptake inhibitor (NRI) properties. ${ }^{13}$ Both antidepressants have different neurochemistry ratios at their starting doses and cannot be seen as equivalent. Using this extrapolative method, perhaps equivalent doses are $150 \mathrm{mg}$ and $50 \mathrm{mg}$, respectively, because venlafaxine ER's NRI potential becomes noticeable at $150 \mathrm{mg} / \mathrm{d}$, likely comparable to desvenlafaxine's $50 \mathrm{mg}$ starting dose.

Pharmacokinetically, we should again cite the work of Nichols et al., ${ }^{4,8}$ and observe the ratios of desvenlafaxine to venlafaxine after metabolism (DES/VEN ratio). Efficient metabolizers taking $75 \mathrm{mg}$ of venlafaxine developed a DES/VEN ratio of 6.2, where poor metabolizers were approximately $50 \%$ less with a 3.3 ratio. Therefore, in some efficient metabolizing patients, $75 \mathrm{mg}$ of parent venlafaxine ER drug may convert to a $300 \mathrm{mg}$ equivalent of desvenlafaxine. As a result, the jury is out, in that $50 \mathrm{mg}$ of desvenlafaxine metabolite antidepressant might equal $75 \mathrm{mg}, 150 \mathrm{mg}$, or $18.75 \mathrm{mg}$ of the parent drug.

The art of psychopharmacology is to analyze the evidence base and data available to us as practitioners and use it appropriately. Where the data end clinicians must theorize and support their practices. In the case of venlafaxine versus desvenlafaxine, there seems to be minimal clinical benefit when comparing a few thousand patients who participated in regulatory studies. In clinical practice, where the sample size is one patient at a time, clinicians oftentimes see the parent drug fail in effectiveness and more so fail in regard to tolerability, only to switch to a new metabolite, isomer, slow release preparation, etc., and titrate farther and ultimately obtain remission. How ever, these types of statistics are often not publicized, seen by pharmaceutical company critics, or appreciated by insurance companies who often refuse more expensive drugs. This editorial, offers some theory in regard to the metabolite desvenlafaxine and why it may work better and be safer in certain, select patients who are not accounted for in regulatory trials or their interpretations.

\section{Disclosures}

The author is a consultant and has received consulting fees from Dey/Mylan and Pamlab. He conducts research for, and received grants from Cephalon/Teva, BristolMyers Squibb, and Cyberonics.

\section{References}

Sequenced Treatment Alternatives to Relieve Depression (STAR*D) Study.

1. National Institute of Mental Health. Sequenced Treatment Alternatives to Relieve Depression (STAR*D) Study. http://www.nimh.nih.gov/trials/practical/ stard/index.shtml. Accessed 5/1/12.

2. CO-MED. Combining Medications to Enhance Depression Outcomes. http://www.co-med.org. Accessed 5/1/12.

3. Preskorn SH. Understanding outliers on the usual dose response curve: venlafaxine as a way to phenotype patients in terms of their CYP 2D6 status and why it matters. J Psychiatr Pract. 2010; 16(1): 46-49.

4. Nichols AL, Focht $\mathrm{K}$, Jiang $\mathrm{O}$, et al. Pharmacokinetics of venlafaxine extended release $75 \mathrm{mg}$ and desvenlafaxine $50 \mathrm{mg}$ in healthy CYP2D6 extensive and poor metabolizers: a randomized, open-label, two-period, parallel-group, crossover study. Clin Drug Investig. 2001; 31(3): 155-167.

5. Lobello KW, Preskorn SH, Guico-Pabia CJ. CYP2D6 phenotype predicts antidepressant efficacy of venlafaxine. J Clin Psychiatry. 2010; 71(11): 1482-1487. 
6. Bachmeier CJ, Beaulieu-Abdelahad D, Ganey NJ, et al. Induction of drug efflux protein expression by venlafaxine but not desvenlafaxine. Biopharm Drug Dispos. 2011; 32(4): 233-244.

7. Lin JH. Drug-drug interaction mediated by inhibition and induction of P-glycoprotein. Adv Drug Deliv Rev. 2003; 55: 53-81.

8. Nichols AI, Tourian KA, Tse SY, et al. Desvenlafaxine for major depressive disorder: incremental clinical benefits from a second-generation serotonin-norepinephrine reuptake inhibitor. Expert Opin Drug Metab Toxicol. 2010; 6(12): 1565-1574.

9. Droll K, Bruce-Mensah K, Otton SV, et al. Comparison of three CYP2D6 probe substrates and genotype in Ghanaians, Chinese and Caucasians. Pharmacogenetics. 1998; 8(4): 325-333.
10. Ieiri I, Takane H, Otsubo K. The MDR1 (ABCB1) gene polymorphism and its clinical implications. Clin Pharmacokinet. 2004; 43(9): 553-576.

11. Guico-Pabia CJ, Jiang Q, Ninan PT, et al. Clinical outcomes following switch from venlafaxine ER to desvenlafaxine in nonresponders and responders. Curr Med Res Opin. 2011; 27(9): 1815-1826.

12. Bech P, Boyer P, Germain JM, et al. HAM-D17 and HAM-D6 sensitivity to change in relation to desvenlafaxine dose and baseline depression severity in major depressive disorder. Pharmacopsychiatry. 2010; 43(7): 271-276.

13. Norman T. The new antidepressants-mechanisms of action. Aust Prescr. 1999; 22: 106-108. 